\title{
Direct determination of cellulosic glucan content in starch- containing samples
}

\author{
Justin B. Sluiter $\cdot$ Katie P. Michel • Bennett Addison • Yining Zeng • \\ William Michener • Alexander L. Paterson • Frédéric A. Perras • \\ Edward J. Wolfrum
}

Received: 30 June 2020/ Accepted: 15 December 2020/Published online: 15 January 2021

(C) This is a U.S. government work and not under copyright protection in the U.S.; foreign copyright protection may apply 2021

\begin{abstract}
A simple and highly selective analytical procedure is presented for the determination of cellulosic glucan content in samples that contain both cellulose and starch. This method eliminates the unacceptably large compounding errors of current two-measurement methods. If both starch and cellulose are present before analytical hydrolysis, both will be hydrolyzed to glucose causing bias and inaccuracy in the method. To prevent this interference, the removal of starch prior to cellulosic quantification is crucial. The method presented here is a concise inseries procedure with minimal measurements, eliminating large compounding errors. Sample preparation consists of a starch extraction employing enzymatic hydrolysis followed by a simple filtration and wash. The samples are then subjected to a two-stage acid hydrolysis. The concentration of glucose is determined by ion exchange high-performance liquid
\end{abstract}

Supplementary Information The online version contains supplementary material available at (https://doi.org/10.1007/ s10570-020-03652-2).

J. B. Sluiter $(\bowtie) \cdot$ K. P. Michel · B. Addison •

Y. Zeng · W. Michener · E. J. Wolfrum

National Renewable Energy Laboratory, Renewable

Resources and Enabling Sciences Center, 15013 Denver

West Parkway, Golden, CO 80401, USA

e-mail: Justin.Sluiter@nrel.gov

A. L. Paterson · F. A. Perras

Ames Laboratory, Chemical and Biological Sciences, 2416 Pammel Dr., Ames, IA 50011, USA chromatography with a $\mathrm{Pb}^{2+}$ column and a refractive index detector. The cellulosic glucan content is calculated based on the initial dry weight of the starting material. Data for the native biomass materials studied show excellent reproducibility, with coefficients of variance of $3.0 \%$ or less associated with the method. This selectivity for cellulosic glucan by the procedure was validated with several analytical techniques such as liquid chromatography coupled with mass spectrometry (LC-MS), Raman spectroscopy, and nuclear magnetic resonance.

Keywords Analytical - Carbohydrate - Cellulose · Glucose $\cdot$ Starch $\cdot$ Biofuels $\cdot$ Ethanol $\cdot$ DNP

\section{Introduction}

Conversion of starch to ethanol is an ancient technology that is still used today at industrial scale to produce renewable ethanol as fuel. Because of its history, this well-established conversion of starch, an $\alpha$ - $(1,4)$-linked glucan carbohydrate, is often referred to as a first-generation technology. Conversion of cellulose, a $\beta$-(1,4)-linked glucan carbohydrate, to ethanol, has yet to be widely commercialized but has been extensively researched (Solomon et al. 2007; Orts and McMahan 2016). This technology is referred to as a second-generation technology. In the last decade, an interesting hybrid of these two conversion 
technologies has evolved where cellulose present in corn grain (the corn kernel fiber) is converted at a firstgeneration plant either in the same fermenter (in situ) as the starch or in a separate fermenter (ex situ) after starch conversion. As this technology is viewed as a step between a first-generation conversion and a second-generation conversion it often called a generation 1.5 process. Current processes to convert biomass material to biofuels remain largely proprietary. For an overview of biomass conversion to produce ethanol please visit the DOE website and review the "Biomass Conversion: From Feedstocks to Final Products" factsheet (DOE 2016).

\section{Background}

There is a need for a publicly accessible, accurate, and specific analytical method for the determination of cellulose in materials containing both starch and cellulose to advance the generation 1.5 biorefinery industry. The Environmental Protection Agency (EPA) Renewable Fuel Standard (RFS) requires a calculation of cellulosic conversion in existing starchto-ethanol fermentation plants for access to valuable financial incentives (EPA 2014, 2019). There are limitations in place as to which analytical methods are allowable for qualification to related subsidies. The regulations place higher value on carbohydrates from non-starch sources, but require analytical verification that the converted carbohydrates originate from nonstarch (i.e. cellulosic) material, and have a preference for analytical methods that have been approved by Voluntary Consensus Standard Body (VCSB) organizations. Current VCSB methods do not directly speciate carbohydrate source in a mixture of starch and cellulose.

The key issue associated with cellulosic glucan quantification in samples that contain significant quantities of starch or free sugars derived from starch is that starch and cellulose are both glucan polymers chemically differentiated only by a single bond orientation. The standard cellulose determination method involves acid hydrolysis of glucan polymers to glucose followed by glucose measurement (AOAC International 2012) and it is impossible to identify the source of glucose once acid hydrolysis has depolymerized the native structures.
To resolve this issue, the current practice is to utilize two analytical methods: (1) acid hydrolysis to determine the total glucose content of the samples, which accesses both starch and cellulose-derived glucose and (2) enzymatic hydrolysis to determine the portion of glucose from starch or starch-derived glucose. The cellulose content is then calculated as the difference between the total glucan content measured in (1) and the starch glucan content measured in (2). There are several concerns with this approach. First, accurate cellulose quantification relies heavily on an accurate starch assay, and in the recent National Institute of Standards and Technology (NIST) publication that documents starch assays for corn grains, there were a variety of assay types reported as being used in the industry. While the NIST document does not specifically correlate the different assays with the variability in the starch quantification, there is disagreement among participant reported starch contents (Murray 2020). Of significant concern is that starch is comprised of both labile and resistant starch moieties, with the resistant starch being defined by resistance to enzymatic degradation (Eerlingen and Delcour 1995; Sajilata et al. 2006). Secondly, due to the different reactivities of the starch and cellulose to the acid hydrolysis, there is likely a significant bias in the calculated cellulose content when substantial amounts of starch or free sugars are present. Finally, the analytical methods currently employed to quantify cellulose and starch are complex and difficult to perform, leading to issues with deployment of the methods to commercial laboratories that would need to implement them for a growing industry.

In this work we present an analytical method that can quantify the cellulosic glucans present in corn grain samples. Cellulosic glucans are comprised of all $\beta$-linked glucans. We do this by first enzymatically hydrolyzing the starch components of the sample and removing the starch derived glucose by washing. The remaining pellet, which contains only cellulosic glucans, is hydrolyzed with a well-known acid hydrolysis procedure which converts the cellulosic glucan to glucose for measurement by high pressure liquid chromatography (HPLC) (Fig. 1). 


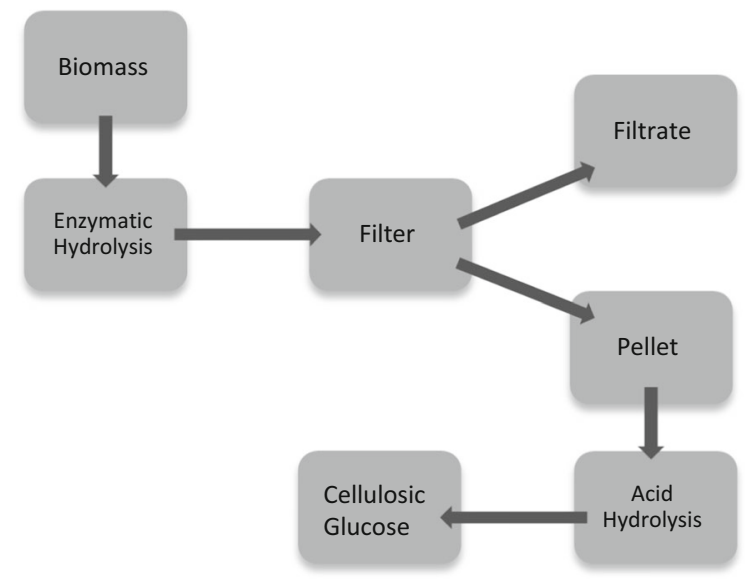

Fig. 1 Flow chart of the analytical procedure

\section{Materials and methods}

Standards

Two native matrices were obtained from NIST to demonstrate the efficiency of this procedure on industrial material: Biomass A, a corn grain flour from a corn ethanol plant and Biomass $\mathrm{B}$, a processed (post-fermentation) corn grain product from a corn ethanol plant. Avicel, purchased from Sigma-Aldrich (St. Louis, MO), was used as a representative pure cellulose material, and a resistant starch (high amylose content), obtained from Ingredion (Westchester, IL), was used as a representative pure starch material. High purity sugar concentration standards were purchased from Absolute Standards Inc. (Hamden, CT), with a concentration range of 0.01-6 mg/mL (Part \#64417).

Reagents and consumables

All chemical reagents used were ACS grade: sodium hydroxide, glacial acetic acid, calcium carbonate, calcium chloride dihydrate and sulfuric acid. All solutions were made using filtered de-ionized water that was obtained from a Milli-Q, EMDMillipore system (Burlington, MA). Sample filtration was performed using quartz fiber filters (cat. No. QR100) that were purchased from Advantec (Dublin, CA). Enzymes for the enzymatic hydrolysis of starch were purchased from Megazyme (Bray, Ireland) thermostable $\alpha$-amylase (product code E-BSTAA) and Amyloglucosidase (AMG) (product code E-AMGDF40ML).

\section{Equipment}

Quantification of glucose was performed using an Agilent (Foster City, CA) 1100 series high-performance liquid chromatography (HPLC) system equipped with a refractive index detector (RID). A Shodex sugar SP0810 column was purchased from Phenomenex (Torrance, $\mathrm{CA}$ ) and an ionic form $\mathrm{H}^{+} /$ $\mathrm{CO}_{3}{ }^{-}$deashing guard column was purchased from Biorad (Hercules, CA). Acid hydrolysis was carried out in an 18 cubic foot autoclave purchased from Consolidated Sterilizer Systems, (Billerica, MA) model name: SSR-24C.

Procedure

\section{Sample preparation}

As specified by the National Renewable Energy Laboratory (NREL) laboratory analytical procedures (LAP), all samples were air-dried to less than $10 \%$ moisture content prior to beginning the analysis. Total moisture contents of dried samples were determined by drying at $105{ }^{\circ} \mathrm{C}$ for at least $8 \mathrm{~h}$ according to NREL LAP "Determination of Total Solids in Biomass and Total Dissolved Solids in Liquid Process Samples" (Sluiter et al. 2008).

\section{Starch and free sugar removal}

The starch and free sugar removal procedure was adapted from a published Megazyme starch assay procedure (McCleary et al. 2019), the rapid total starch (RTS)-NaOH method. $200 \pm 10 \mathrm{mg}$ of dried sample was weighed out into a polypropylene $50 \mathrm{~mL}$ conical centrifuge tube. $0.4 \mathrm{~mL}$ of 190 proof ethanol was added to the tube while vortexing to aid in sample dispersion (as described in AOAC 996.11 (AOAC International 2012). Then $4 \mathrm{~mL}$ of a cold $1.7 \mathrm{M} \mathrm{NaOH}$ solution was added and vigorously vortexed for no less than $15 \mathrm{~s}$. Placing the tube on a magnetic stir plate with a stir bar added (VWR cat. No. 89030-550), the samples were stirred for $15 \mathrm{~min}$ with intermittent vortexing throughout (2-3 times). The sodium hydroxide acts as a chaotropic agent, weakening the hydrophobic effect of proteins, allowing the $\alpha$-specific enzymes to gain better access to resistant starch bonds and aiding in breakdown. 
Then $16 \mathrm{~mL}$ of $600 \mathrm{mM}$ sodium acetate buffer containing $5 \mathrm{mM}$ sodium dichloride stabilizer at a $\mathrm{pH}$ of 3.8 was added, well to ensure the final sample $\mathrm{pH}$ is around 5 so that enzyme activity is not affected. Immediately $0.2 \mathrm{~mL}$ of $\alpha$-amylase was added, followed by $0.2 \mathrm{~mL}$ of AMG. The tube was capped, vortexed for $3 \mathrm{~s}$, and placed into a $50{ }^{\circ} \mathrm{C}$ water bath for $1 \mathrm{~h}$, vortexing the sample halfway through incubation. Once removed from the water bath, the samples were cooled to room temperature prior to filtration.

To separate the starch and free sugar derived glucose solution from the cellulosic solid in the mixture, the samples were then vacuum filtered through a quartz fiber filter. The undiluted filtrate can then be analyzed for glucose and other carbohydrates by HPLC, if desired (this method has not been optimized for this analysis). The solid material was quantitatively transferred from the sample tube to the quartz fiber filter with a series of rinses to ensure complete material transfer, with a minimum of $30 \mathrm{~mL}$ of water used to rinse the sample tubes and solid material. The residual cellulosic solid material and quartz fiber filter, hereafter referred to as the pellet, were then transferred to a $40{ }^{\circ} \mathrm{C}$ vacuum oven to dry overnight.

\section{Acid hydrolysis of cellulosic carbohydrates}

After the starch-derived carbohydrates have been removed, the samples undergo a two-stage acid hydrolysis, first with concentrated sulfuric acid at $30{ }^{\circ} \mathrm{C}$ followed by a dilute acid hydrolysis at high temperature and pressure. The standard LAP "Determination of Structural Carbohydrates and Lignin in Biomass" (Sluiter et al. 2012) was used with minor adaptations. To meet minimum detection and quantification limits on the HPLC, the first and second stage acid hydrolysis volumes were altered to accommodate the anticipated final concentration of cellulosic glucan. Any "low" cellulose sample with less than $3 \%$ cellulose (such as Biomass A) had the acid hydrolysis performed at $1 / 3$ the scale described in the LAP, and any "high" cellulose sample with more than $3 \%$ cellulose (such as Biomass B) had the acid hydrolysis performed at 2/3 the normal scale (Table 1). Any material that has an unknown concentration of cellulose should be analyzed as if it were a low concentration cellulose sample.
The pellet was placed into a $16 \times 100 \mathrm{~mm}$ glass culture tube and the correct corresponding volume of $72 \%(\mathrm{w} / \mathrm{w})$ sulfuric acid was added to each tube. Using a Teflon stir rod, the pellet was intermittently kneaded approximately every $5 \mathrm{~min}$ during the $1 \mathrm{~h}$ incubation period while suspended in a $30{ }^{\circ} \mathrm{C}$ water bath. The sample tubes were then removed from the water bath. The concentration of sulfuric acid was adjusted to $4 \%$ by adding the appropriate volume of filtered deionized (DI) water split into multiple aliquots, which were used to rinse the macerated pellet into a pressure tube (Ace Glass \#8648-89), quantitatively transferring the contents of the culture tube into each pressure tube. The pressure tubes were then capped and placed in an autoclave and kept at $121{ }^{\circ} \mathrm{C}$ for $1 \mathrm{~h}$ on a liquids cycle. Alongside the samples, acidified sugar recovery standards (SRS) were also autoclaved to account for sugar degradation. The SRS are run in triplicate with a glucose concentration of $5 \mathrm{mg} / \mathrm{mL}$, having a $10 \mathrm{~mL}$ standard volume with $348 \mu \mathrm{L}$ of $72 \%$ (w/w) sulfuric acid added. Prior to the autoclave step, the acidified SRS are divided in half; one half to be autoclaved for post-hydrolysis concentration determination and the other half for analysis of initial starting concentration.

Once cooled from the autoclave, the samples and SRS were neutralized using calcium carbonate to a $\mathrm{pH}$ of approximately 5-7 (the working range of the HPLC column), and passed through a $0.2 \mu \mathrm{m}$ nylon syringe filter (MDI Membrane Technologies, cat. No. SYGN) into autosampler vials for HPLC analysis. Calcium carbonate was specifically chosen to protect the longevity of the Shodex sugar SP0810 column, as calcium sulfate, the salt which forms during the neutralization, is largely insoluble and prevents excess cations from altering the column chemistry.

\section{HPLC analysis}

A five-point calibration curve with the concentration range of $0.01-6 \mathrm{mg} / \mathrm{mL}$ was analyzed prior to samples. Analytes included in the purchased standards were: D-cellobiose, $\mathrm{D}(+)$-glucose, $\mathrm{D}(+)$-xylose, $\mathrm{D}(+)$ galactose, $\mathrm{L}(+)$-arabinose, $\mathrm{D}(+)$-mannose. A calibration verification standard (CVS) with a concentration in the middle of the calibration curve was analyzed immediately following the calibration curve and approximately every tenth injection throughout the HPLC sequence, to ensure instrument accuracy (Table 2). 
Table 1 Volumes for two-stage acid hydrolysis, *volumes adjusted for densities

\begin{tabular}{llll}
\hline $\begin{array}{l}\text { Anticipated cellulose concentration in } \\
\text { biomass }(\%)\end{array}$ & $\begin{array}{l}\text { Volume of } 72 \%(\mathrm{w} / \mathrm{w}) \\
\mathrm{H}_{2} \mathrm{SO}_{4}(\mathrm{~mL})\end{array}$ & $\begin{array}{l}\text { Volume of filtered } \mathrm{DI} \mathrm{H}_{2} \mathrm{O} \text { for } \\
\mathrm{H}_{2} \mathrm{SO}_{4}(\mathrm{~mL})\end{array}$ & $\begin{array}{l}\text { Final volume of } \\
\text { hydrolysis* }\end{array}$ \\
\hline Low $(0-3)$ & 1 & 28 & 28.91 \\
High $(>3)$ & 2 & 56 & 57.82 \\
\hline
\end{tabular}

Table 2 HPLC operating conditions

\begin{tabular}{ll}
\hline Method acquisition parameter & Setting specification \\
\hline Injection volume & $50 \mathrm{~mL}$ \\
Mobile phase & $\mathrm{HPLC}$ grade water, $0.2 \mu \mathrm{m}$ filtered and degassed \\
Flow rate & $0.6 \mathrm{~mL} / \mathrm{min}$ \\
Autosampler temperature & $4{ }^{\circ} \mathrm{C}$ \\
Guard cartridge temperature & Ambient \\
Column temperature & $85^{\circ} \mathrm{C}$ \\
Detector temperature & $55^{\circ} \mathrm{C}$ (as close to column temperature as possible) \\
Detector & Refractive index \\
Run time & 35 min data collection plus 7 min post run \\
\hline
\end{tabular}

\section{Calculations}

The concentration of monomeric sugars in solution was calculated using a linear regression calibration curve created using Agilent Chemstation software. This measured concentration was corrected for the average amount of sugar recovered from triplicate SRS to obtain the pre-hydrolysis glucose concentration $\left(\mathrm{C}_{\mathrm{x}}\right)$. Using the oven dried weight of the starting sample material $\left(\mathrm{M}_{\mathrm{x}}\right)$, the final volume of hydrolysis $(\mathrm{V})$, and the stoichiometric factor between glucose and glucan (A): 162/180, the percentage of the glucan originating from cellulosic material can be calculated via Eq. (1).

$\%$ Cellulosic Glucan $=\frac{C_{x} \times V \times A}{M_{x}}$

\section{Results and discussion}

Validation of method specificity

In this method, we rely on enzymatic hydrolysis to quantitatively remove all starch from the sample while retaining cellulosic material prior to the analytical hydrolysis procedure. The presence of any starch after the first procedure will bias the cellulosic measurement high in the second procedure. Conversely, the presence of any cellulosic-derived carbohydrates in the filtrate will result in a low bias. To validate the efficacy of the enzymatic hydrolysis to remove all starch while retaining all cellulosic material, various advanced analytical techniques were utilized to detect the presence or absence of both cellulosic glucan in destarching filtrate and alpha linked glucans in the destarched pellet. The following section will discuss the experimental conditions and results obtained from the analyses of the treated (de-starched) and untreated (as received) biomass materials, as well as the filtrate liquid after enzymatic hydrolysis, as compared to representative reference materials. Techniques used include liquid chromatography-mass spectrometry (LC-MS), Raman, and nuclear magnetic resonance spectroscopy (NMR).

\section{Analysis of filtrate for evidence of soluble cellulosic material}

Common cellulose solubilization techniques involve alkaline conditions at various concentrations and temperatures (Knill and Kennedy 2003). Thus, to investigate whether the sodium hydroxide pre-treatment of the sample prior to enzymatic hydrolysis 
partially solubilized any significant amount of cellulosic material, we looked for the presence of cellobiose in the filtrate after pellet filtration. Cellobiose, the $\beta$-(1,4)-linked glucan dimer, was chosen as the smallest molecule that retains the linkage indicative of cellulose solubilization; any detectable cellobiose would indicate cellulose loss during enzymatic hydrolysis.

The samples were injected at $10 \mu \mathrm{L}$ per run, separated and detected using an Acquity Ultra Performance Liquid Chromatography (UPLC) system (Waters Co., Milford, MA) equipped with an Evaporative Light Scattering Detector (ELSD) and Waters TQD (Waters Co., Milford, MA) mass spectrometer. The chromatography system used a Shodex Sugar SZ5532(Zinc) column (Showa Denko K.K., Japan) at $6 \times 150(\mathrm{~mm}), 6 \mu \mathrm{m}$ particle size, to sufficiently separate the dimers of interest. See supplementary information section LC-MS for specific acquisition parameters. The masses examined by the experiment were $[\mathrm{M}-\mathrm{H}]^{-}$of $341 \mathrm{~m} / \mathrm{z},[\mathrm{M}-\mathrm{H}+\mathrm{FA}]^{-}$of $387 \mathrm{~m} / \mathrm{z}$ and $[\mathrm{M}-\mathrm{H}+\mathrm{NaFA}]^{-} 409 \mathrm{~m} / \mathrm{z}$.

A standard solution showed a retention time for cellobiose at approximately $19.8 \mathrm{~min}$. The filtrates for both treated Biomass A and Biomass B had peaks on the ELSD detector at or around 10.6 min (Fig. 2a) which matched with a glucose standard solution (not shown). Examination of the mass spectra for cellobiose at the retention time in the filtrates showed that cellobiose was not detected at that specific retention time of $19.8 \mathrm{~min}$. Other peaks with mass of $341 \mathrm{~m} / \mathrm{z}$ and $387 \mathrm{~m} / \mathrm{z}$ were detected in samples Biomass A and Biomass $\mathrm{B}$ at different retention times then cellobiose. It is likely that these ions are associated with maltodextrins, but we were not able to confirm this identification.

Daughter ion experiments for a cellobiose standard and the biomass filtrate samples were performed. The cellobiose standard did not form a sodium formate adduct but did form the other two adducts of $[\mathrm{M}-\mathrm{H}]^{-}$ $(341 \mathrm{~m} / \mathrm{z})$ and $[\mathrm{M}-\mathrm{H}+\mathrm{FA}]^{-}(387 \mathrm{~m} / \mathrm{z})$. In this experiment, the cellobiose retention time for ions 341 and $387 \mathrm{~m} / \mathrm{z}$ was approximately $18.70 \mathrm{~min}$. For this experiment, no ions were detected for any adducts for cellobiose in the filtrate of Biomass A or Biomass $\mathrm{B}$ at this retention time. The evaluation of the ions detected and comparisons to cellobiose mass spectra are included in the supplemental information. The exact identities of the peaks in the filtrates is currently
Fig. 2 a Chromatograms for a cellobiose standard, filtrate from Biomass A, and filtrate from Biomass B; ELSD response scaled to maximum peak height. $\mathbf{b}$ Mass spectra of the peak of interest at $19.8 \mathrm{~min}$

unknown, but we conclude that there is no detectable cellobiose present in the filtrate solutions.

\section{Analysis of pellet for evidence of remaining starch}

The starch removal procedure selected should be aggressive enough to remove all labile and resistant starch present. It has been reported that various types of resistant starch remain after fermentation in dried distiller grains ( $\mathrm{Li}$ et al. 2014; Srichuwong and Jane 2011). To verify that this procedure is aggressive enough for the removal of all labile and resistant starch present, Raman and NMR spectroscopy were used to verify the absence of starch in the pellet. Treated and untreated biomass solid materials were compared to pure resistant starch and pure cellulose material to qualitatively show the removal of starch, and the continued presence of cellulosic material.

The Raman spectroscopy data indicated qualitatively that the starch removal was successful as there was an overall loss of bands in the starch specific regions. Unfortunately, this application was not robust enough for definitive validation, as the removal of starch resulted in a material with a high fluorescent background, likely a result of increased aromatics concentration due to starch removal. This required extensive use of photobleaching for noise reduction (Butler et al. 2016) that resulted in spectra that were greatly reduced in intensity and difficult to compare to the starting biomass material. For completeness, we have included the details of this work (including the specific method used) as supporting information.

${ }^{13} \mathrm{C}$ cross-polarization magic-angle-spinning (CPMAS) solid-state NMR data shown in Fig. 3 (and S5) reveals direct evidence that the enzymatic treatment procedure removes starch carbohydrates but leaves cellulosic material intact. Based on the intensities of the characteristic cellulose signals at $89 \mathrm{ppm}$ and $105 \mathrm{ppm}$, cellulose appears intact post treatment. Interestingly, after treatment we see a higher relative protein content (aliphatics, carbonyl) compared to carbohydrate content. This is likely because as the carbohydrates (starch) are removed, protein content is 
a

\#2 Biomass B

201016 Cellobiose 1 Test

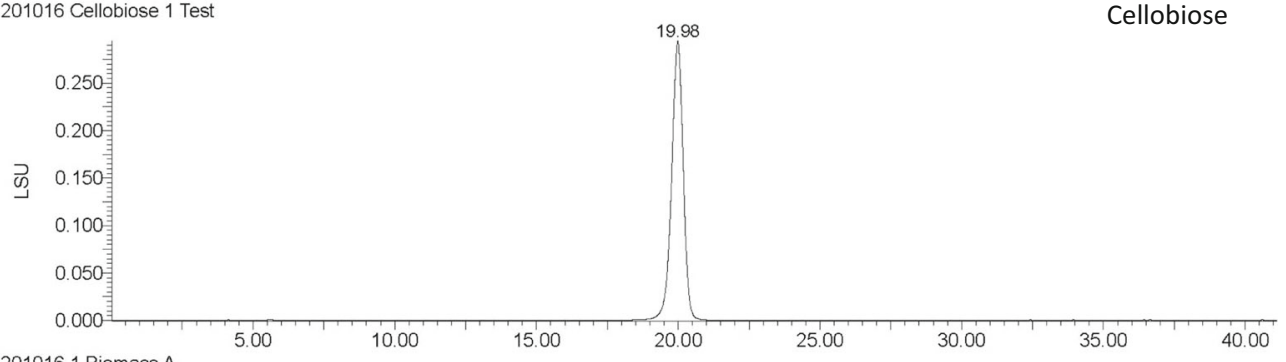

2010161 Biomass A

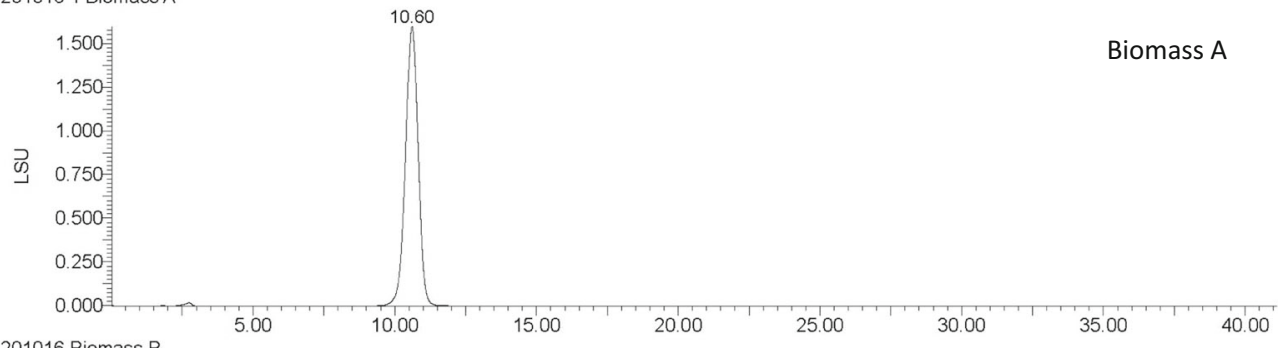

201016 Biomass B

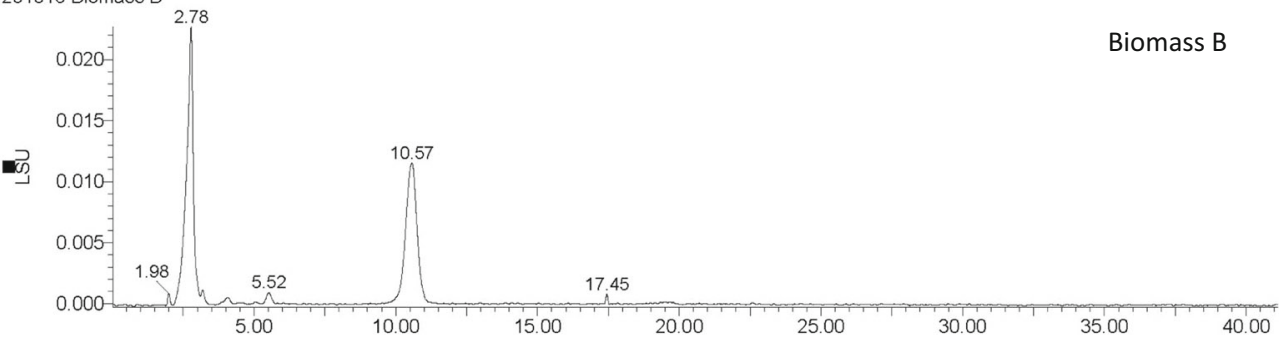

b
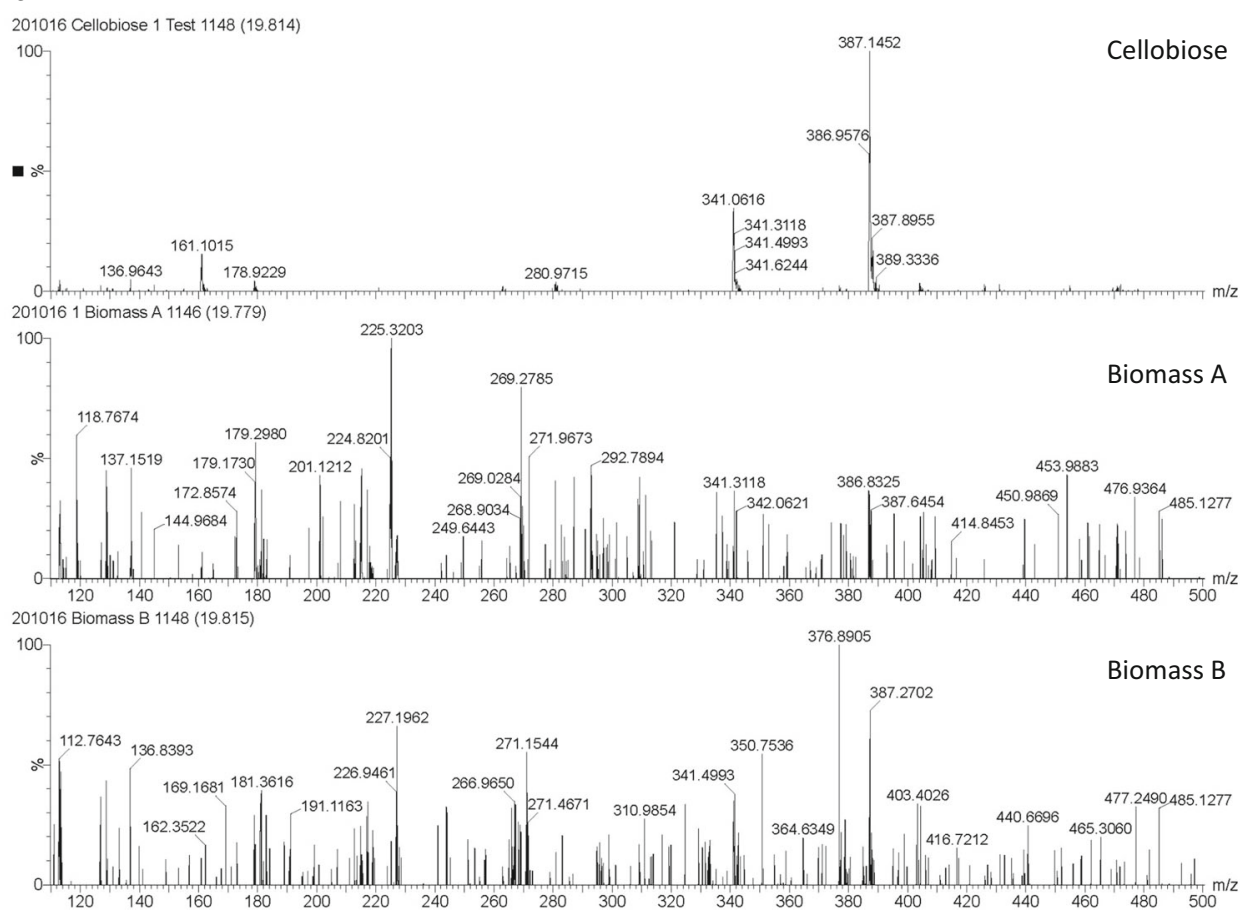


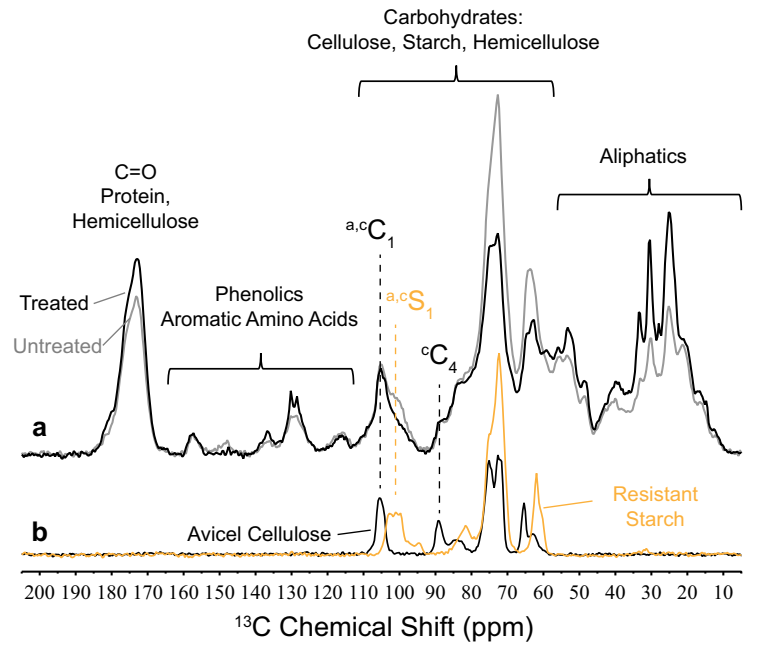

Fig. 3 (a) ${ }^{1} \mathrm{H}_{-}{ }^{13} \mathrm{C}$ CP-MAS NMR $\left(150 \mathrm{MHz}{ }^{13} \mathrm{C}\right.$ Lamor frequency) Biomass $\mathrm{B}$, letters $\mathrm{C}$ and $\mathrm{S}$ refer to cellulose and starch, superscripts a and c refer to amorphous and crystalline allomorphs, and superscript numbers refer to carbon position, biomass spectra in (a) were scaled by mass

largely unchanged, resulting in a higher protein to carbohydrate ratio after treatment. The signature anomeric carbon region in the 100-105 ppm range clearly shows a loss of a broad resonance centered near 100-102 ppm, assigned to the starch anomeric $\mathrm{S}_{1}$ carbon. The remaining shoulder near 100-102 ppm after treatment most likely arises from hemicelluloses, but we cannot rule out residual starch from the CPMAS data alone. The interpretation that the starch is fully removed and that the residual shoulder is assigned to other carbohydrates (e.g. glucuronoxylan) was confirmed using Dynamic Nuclear Polarization (DNP)-enhanced MAS NMR measurements (Fig. 4) and gel-state NMR methods (Figure S9). Also, based on the intensities of the characteristic cellulose signals at $89 \mathrm{ppm}$ and $105 \mathrm{ppm}$, cellulosic material appears intact post treatment.

Carbon-carbon connectivity information for treated and untreated biomass samples, as well as starch and cellulose control materials, was extracted using the through-bond ${ }^{13} \mathrm{C}_{-}{ }^{13} \mathrm{C}$ single-quantum (SQ) double-quantum (DQ) ${ }^{13} \mathrm{C}$ refocused INADEQUATE (Incredible Natural Abundance DoublE QUAntum Transfer Experiment) (Bax et al. 1981; Lesage et al. 1999) parameters. Note that the DQ shifts correspond to the sum of the chemical shifts of the two correlated sites; this greatly helps resolve overlapping signals in the indirect dimension. Performing such an

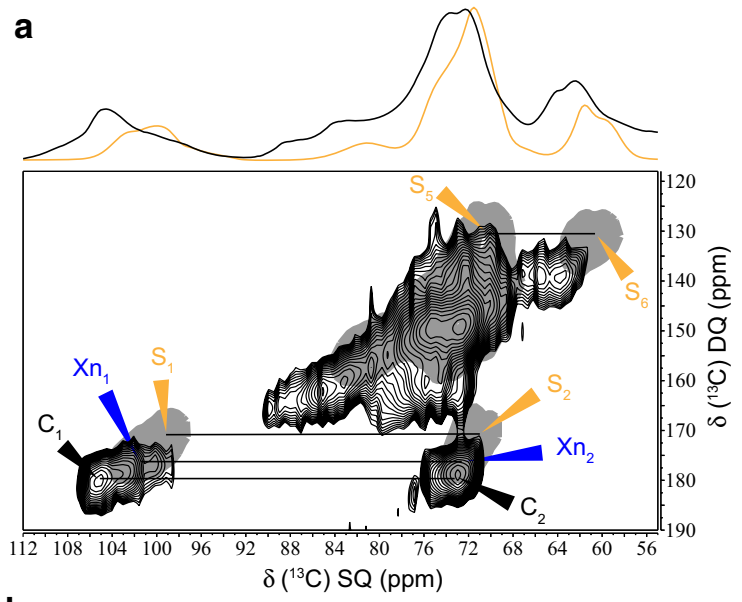

b

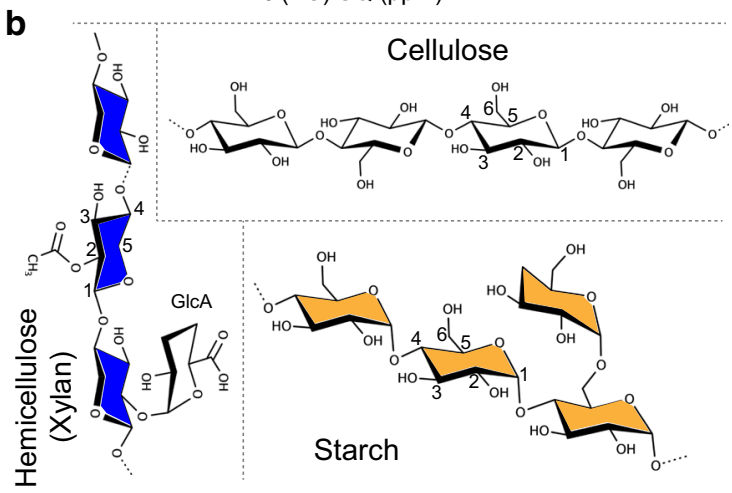

Fig. 4 DNP-enhanced ${ }^{13} \mathrm{C}_{-}{ }^{13} \mathrm{C}$ refocused SQ/DQ refocused INADEQUATE data on treated Biomass B (black contours) is overlaid with resistant starch (solid grey) at natural abundance, relevant assignments for starch, xylan and cellulose are indicated with corresponding chemical structures shown in $\mathbf{b}$

experiment at natural abundance $\left(1.1 \%\right.$ for $\left.{ }^{13} \mathrm{C}\right)$, however, requires significant sensitivity enhancement given that the probability of finding a bonded ${ }^{13} \mathrm{C}$ pair is roughly $0.01 \%$. We have thus turned to DNP to enhance the sensitivity (Maly et al. 2008; Barnes et al. 2008; Slichter 2014). DNP has been used a number of times to perform ${ }^{13} \mathrm{C}$ correlation experiments on biomass at natural abundance (Rossini et al. 2012; Takahashi et al. 2012; Perras et al. 2017; Kirui et al. 2019; Kang et al. 2019; Viger-Gravel et al. 2019). As can be seen in Figure S10, DNP worked very well for all samples, yielding enhancement factors in the range of 18 for Avicel cellulose, 78 for resistant starch, and an overall enhancement of 48-64 for biomass samples, corresponding to experiment acceleration factors of 2300-4100.

Figure 4a displays the carbohydrate "fingerprint" region (110 to $60 \mathrm{ppm}$ in the SQ dimension, 120 to 
$190 \mathrm{ppm}$ in the DQ dimension) for DNP-enhanced ${ }^{13} \mathrm{C}$ INADEQUATE spectra of resistant starch (solid grey) and treated Biomass B (black contours). Relevant assignments for cellulose, hemicellulose (xylan) and starch are indicated with $\mathrm{C}, \mathrm{Xn}$ and $\mathrm{S}$ characters, respectively, with subscripts for carbon position. The absence of starch after treatment is clear. Specifically, well-resolved and characteristic correlations for starch, $S_{1}-S_{2}$ (DQ frequency near $170 \mathrm{ppm}$ ) and $\mathrm{S}_{5}-$ $\mathrm{S}_{6}$ (DQ frequency near $131 \mathrm{ppm}$ ), are not detectible in the treated biomass. It is worth noting that the DNP enhancement factor for starch is much larger than for cellulose due to differences in impregnability of the AMUPol polarizing agent and differences in the domain sizes, so if any starch is present the signal should be easily visible in a cellulose background. Moreover, the lower-frequency shoulder in question (SQ signal at $\sim 101-102 \mathrm{ppm}$ ) is predominantly seen in the DQ dimension at $176 \mathrm{ppm}$. This exactly matches the SQ and DQ frequencies for the C1-C2 correlation expected for threefold helical screw xylan as published previously (Busse-Wicher et al. 2014; Dupree et al. 2015; Simmons et al. 2016; Kang et al. 2019, 2020; Zhao et al. 2020).

Further supporting DNP solid-state NMR data, which is capable of observing all carbons present in the pellet, solution-state ${ }^{1} \mathrm{H}_{-}{ }^{13} \mathrm{C}$ HSQC data on ballmilled biomass samples swollen in the gel phase (Figure S9) also clearly show no evidence of starch in the treated samples. The Gel-HSQC data highlights solvent-accessible moieties, which enhances starch signals and suppresses those from cellulose. Taken together the solid-state and solution-state NMR results unequivocally demonstrate that no detectable starch remains in the biomass material after treatment, and also that the residual shoulder can be assigned to hemicellulose polysacharides like disordered xylan. See NMR section of the supplementary information for all NMR acquisition parameters and methods.

Given that we could not find cellobiose present in the filtrate, which would indicate loss of cellulose to the liquid phase, we conclude that all cellulosic glucan remains in the pellet after starch removal. Extensive analysis of the pellet for the presence of starch glucan similarly shows no evidence of contamination of the pellet with $\alpha$-(1,4)-linked glucan carbohydrates. Most notably, solid-state CP-MAS NMR of the pellets show that starch is removed while cellulose and protein content is largely unchanged, while $2 \mathrm{D}{ }^{13} \mathrm{C}-{ }^{13} \mathrm{C}$ refocused INADEQUATE data with DNP enhancement, together with gel-state HSQC data, further support starch removal and provide convincing evidence that no residual starch can be detected in the pellet post treatment. We therefore conclude that the enzymatic hydrolysis can accurately separate the $\alpha$ $(1,4)$-linked glucan carbohydrates from the $\beta$-linked glucan carbohydrates.

\section{Hemicellulose}

Unfortunately, a partial loss of hemicellulose into the sodium hydroxide solution makes this procedure incompatible with a direct measurement of hemicellulose. It had been a secondary objective of this method to simultaneously quantify the carbohydrates associated with hemicellulose. While investigating the possibility of cellulose solubilization into the filtrate, significant quantities of hemicellulose carbohydrates were detected. It has been previously reported that the sodium hydroxide concentrations used during the starch removal process are aggressive enough to solubilize a portion of the hemicellulose present (Cheng et al. 2010). This partial solubilization was verified by acid hydrolysis of the undiluted filtrate and HPLC analysis of the hydrolysate. The comparison of the un-hydrolyzed filtrate and hydrolyzed filtrate shows that a large portion of oligomeric xylan is solubilized. While the purpose of this method was not to optimize conditions for complete dissolution of hemicellulose or cellulose, it is not surprising that a fraction of the hemicellulose would be solubilized given previous work (Isogai and Atalla 1998; Qi et al. 2008).

\section{$\beta$-Glucans}

We recognize that this method cannot differentiate between $\beta$ - $(1,4)$ glucans and other $\beta$-glucans present. In particular, the measurement of cellulose $\beta$-glucans in post-fermentation material will be biased high due to $\beta$-(1,3)-glucans and $\beta$-(1,6)-glucans present in fermentation yeast. An attempt to quantify $b-(1,3)$ glucans present in the post fermentation (DDGS) sample using commercially available enzymatic assay (Megazyme K-EBHLG) for yeast glucans showed significant cross-activity with b- $(1,4)$ cellulose glucan, in line with the manufacturer's data. We have included in the supplementary material the results of the 
enzymatic assay (Table $\mathrm{S} 1$ ) that shows a significant false positive with Avicel microcrystalline cellulose (6\%) and Biomass A (5\%) along with supporting discussion. This measurement is of similar magnitude to that of pure yeast after starch removal treatment $(4 \%)$. We have included in the supplemental information, NMR spectra to support the absence of $\beta-(1,3)$ glucans in the materials used for this assay.

It is apparent that for Avicel cellulose and Biomass A there is no indication of b-(1,3) glucan present, accepting that the commercial enzymatic assay gives rise to a false positive based on hydrolysis of 1,4-bglucan as discussed above.

We conclude that there is not sufficient specificity in the enzyme assays to differentiate these two moieties of glucan. Therefore, the measurement of total cellulose $\beta$-glucans in the post fermented material will be biased high due to the amount of yeast present after the fermentation process, and we could not quantify the percentage of the $b-(1,3)$ and $b-(1,6)$ or mixed linkage glucans still present in the pellet after starch removal treatment. Until further research can be performed in this area, we are unable to address this bias.

\section{Precision and accuracy}

To determine the accuracy for this method we used a known addition approach. In order to best evaluate matrix effects, we spiked known amounts of representative pure chemical compounds into Biomass A and Biomass B. Avicel cellulose was chosen to represent corn grain cellulose, and a resistant starch from maize grain was chosen to represent resistant starch. Each sample of biomass and accompanying spike were individually weighed and analyzed in its entirety to avoid homogeneity concerns. Replicate analysis of each sample type was used for evaluation of precision of the method. Table 3 shows the various spiking levels and replicates analyzed to determine accuracy and precision.

The spiking study was performed by two separate analysts over the course of multiple days on discrete separate batches to incorporate as much environmental variation as possible. The different spike levels were specifically chosen to evaluate a working range: $0.5 \%$ for accuracy close to limit of detection, $1 \%$ to represent an expected working range for samples in production facilities, $10 \%$ for an excessively high level. The calculated mean value, standard deviation, and coefficient of variance $(\mathrm{CV})$ are displayed in Table 3 for each spike level. The simple estimates values were generated using traditional statistical techniques that evaluate the data as a single population without parsing the contribution of the analyst or batch to batch variability.

A linear mixed-effects regression model was generated for the measured cellulosic glucan correlation with the biomass sample, the analyst that performed the work, and the individual batches. These standard deviation values are smaller because the variations which can be attributed to the individual analysts and between batches has been mathematically removed. The simple estimates represent daily variability experienced in a laboratory while the mixed effects model represent the variability attributed solely to the method. The calculated CV values of either assessment of the data satisfactorily meet the objectives for precision.

A linear mixed-effects model was also used to evaluate the ability of the analytic method to quantitatively measure added reference components accounting for differences among analysts and experiments. Some Avicel cellulose spiked groups in Table 4 do not include the expected concentration in the confidence interval (highlighted in bold). All such cases show low recovery. An analytical method with perfect correlation between expected and measured glucan should have a confidence interval that includes the spike level of material added to the sample for all cellulose spike samples and a confidence interval that includes zero for all starch spike samples. Additionally, one starch spike level has a small high bias for the $10 \%$ starch spike level.

Figure 5 is a plot of all cellulosic glucan data generated in the study and clearly shows a strong positive correlation between the amount of Avicel cellulose added to the biomass and an extremely small correlation of measured cellulosic glucan to added resistant starch. Despite a statistically significant difference of measured concentrations from expected concentrations, we see a $93 \%$ recovery of cellulose spiked into the samples. This is consistent with the recoveries seen with neat Avicel cellulose (data not shown). 
Table 3 Calculated means, standard deviations, and coefficient of variance for the measurement of cellulose fraction from spiking experiments using Biomass A and B

\begin{tabular}{|c|c|c|c|c|c|c|c|c|}
\hline \multirow[t]{2}{*}{ Spike } & \multirow[t]{2}{*}{ Level (wt\%) } & \multirow[t]{2}{*}{$\mathrm{N}$} & \multicolumn{3}{|c|}{ Simple estimates } & \multicolumn{3}{|c|}{ Mixed effect regression model } \\
\hline & & & Mean (wt\%) & SD & $\mathrm{CV}(\%)$ & Mean $(w t \%)$ & SD & $\mathrm{CV}(\%)$ \\
\hline \multicolumn{9}{|l|}{ Biomass A } \\
\hline No spike & 0 & 18 & 1.95 & 0.13 & 6.5 & 1.9 & 0.04 & 2.1 \\
\hline Avicel cellulose & 0.5 & 9 & 2.34 & 0.09 & 3.7 & 2.4 & 0.04 & 1.7 \\
\hline Avicel cellulose & 1 & 5 & 3.03 & 0.09 & 3.1 & 2.9 & 0.04 & 1.5 \\
\hline Avicel cellulose & 10 & 5 & 11.34 & 0.34 & 3.0 & 11.2 & 0.11 & 1.0 \\
\hline Resistant starch & 0.5 & 9 & 2.02 & 0.11 & 5.7 & 2.0 & 0.06 & 3.0 \\
\hline Resistant starch & 1 & 5 & 1.97 & 0.14 & 7.1 & 2.0 & 0.06 & 2.8 \\
\hline Resistant starch & 10 & 5 & 2.22 & 0.07 & 3.1 & 2.2 & 0.04 & 1.8 \\
\hline \multicolumn{9}{|l|}{ Biomass $B$} \\
\hline No spike & 0 & 16 & 5.21 & 0.06 & 1.2 & 5.2 & 0.038 & 0.7 \\
\hline Avicel cellulose & 0.5 & 9 & 5.63 & 0.17 & 3.0 & 5.7 & 0.039 & 0.7 \\
\hline Avicel Cellulose & 1 & 5 & 6.32 & 0.17 & 2.7 & 6.1 & 0.040 & 0.7 \\
\hline Avicel cellulose & 10 & 6 & 14.69 & 0.50 & 3.4 & 14.8 & 0.115 & 0.8 \\
\hline Resistant starch & 0.5 & 9 & 5.17 & 0.10 & 1.9 & 5.2 & 0.058 & 1.1 \\
\hline Resistant starch & 1 & 5 & 5.14 & 0.13 & 2.5 & 5.2 & 0.056 & 1.1 \\
\hline Resistant starch & 10 & 5 & 5.21 & 0.05 & 1.0 & 5.2 & 0.039 & 0.8 \\
\hline
\end{tabular}

Table 4 The means and confidence intervals for sample groups analyzed to determine accuracy of the method, concentration of spike measured value is the measured increase in cellulosic glucan from each spike level

\begin{tabular}{|c|c|c|c|}
\hline Spike & Starch spike (wt\%) & Cellulose spike (wt\%) & wt $\%$ of Concentration of Spike Measured $( \pm 95 \% \mathrm{CI})$ \\
\hline \multicolumn{4}{|l|}{ Biomass A } \\
\hline No spike & 0 & 0 & $-0.02(-0.09,0.05)$ \\
\hline Avicel cellulose & 0 & 0.5 & $0.44(0.37,0.52)$ \\
\hline Avicel cellulose & 0 & 1 & $0.91(0.83,0.98)$ \\
\hline Avicel cellulose & 0 & 10 & $9.28(9.06,9.49)$ \\
\hline Resistant starch & 0.5 & 0 & $0.02(-0.10,0.11)$ \\
\hline Resistant starch & 1 & 0 & $0.03(-0.08,0.12)$ \\
\hline Resistant starch & 10 & $\mathbf{0}$ & $0.27(0.20,0.35)$ \\
\hline \multicolumn{4}{|l|}{ Biomass $B$} \\
\hline No spike & 0 & 0 & $-0.04(-0.11,0.03)$ \\
\hline Avicel cellulose & 0 & 0.5 & $0.44(0.37,0.52)$ \\
\hline Avicel cellulose & 0 & 1 & $0.92(0.84,1.00)$ \\
\hline Avicel cellulose & 0 & 10 & $9.54(9.29,9.77)$ \\
\hline Resistant starch & 0.5 & 0 & $-0.01(-0.12,0.10)$ \\
\hline Resistant starch & 1 & 0 & $-0.01(-0.11,0.10)$ \\
\hline Resistant starch & 10 & 0 & $0.00(-0.08,0.08)$ \\
\hline
\end{tabular}




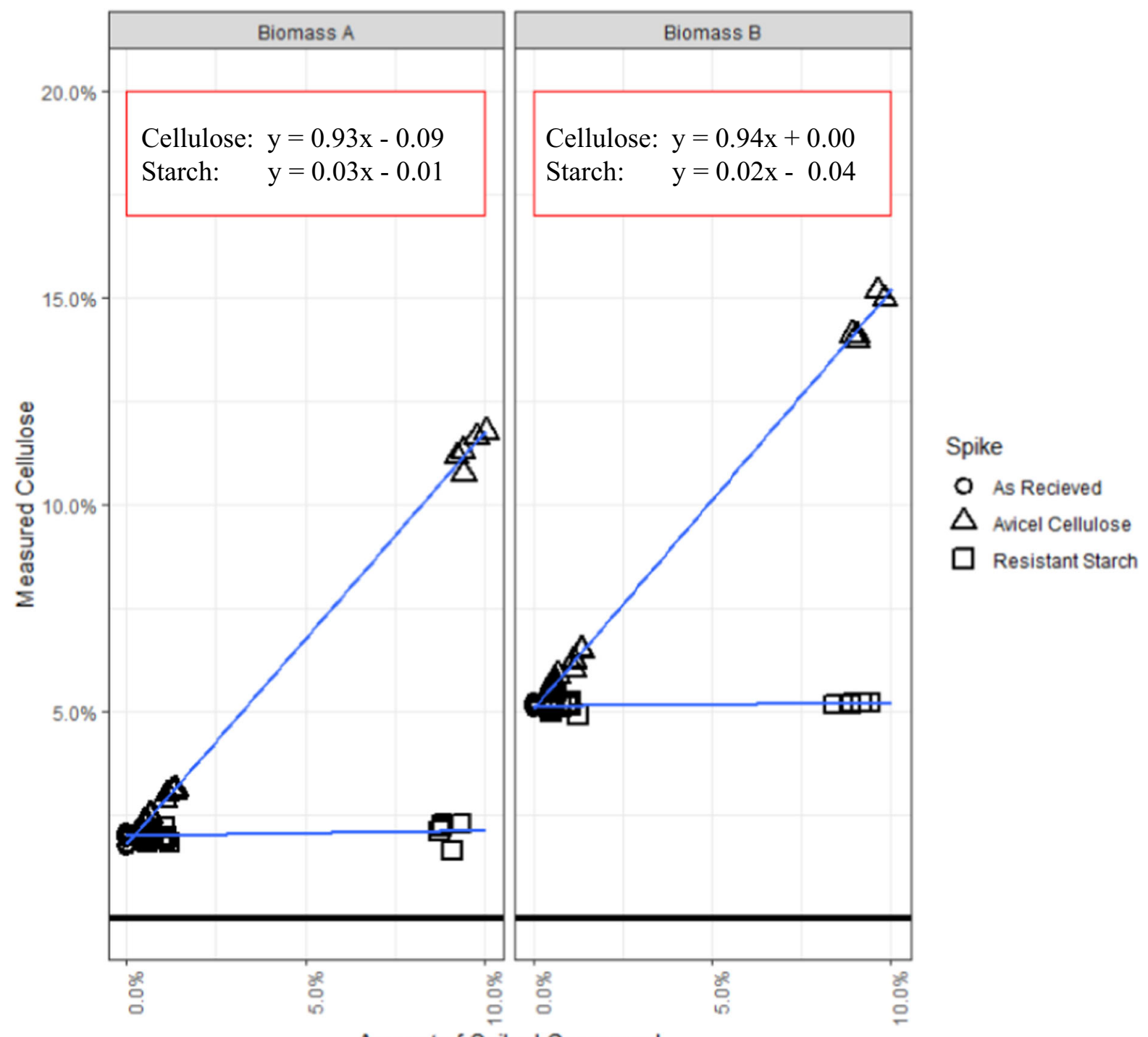

Fig. 5 A plot of expected versus measured cellulose concentration obtained from the spiking study for each biomass material

\section{Conclusion}

This work proposes a method for the measurement of cellulosic glucan in samples containing both cellulose and starch. The method works well as a direct measurement assay for the cellulosic glucan content in samples containing both small and large amounts of starch. The method is specific for cellulosic glucan. When following the procedure, no starch is falsely quantified in the final cellulosic measurement contributing to a high biased result, and no cellulosic material is lost into the filtrate which would cause a low biased final measurement. This is a concise, inseries procedure with minimal measurements which eliminates large compounding errors. The precision and accuracy obtained using this method demonstrate that it is capable of accurate reproducible results.

Acknowledgments We thank Dr. Jacolin Murray at the National Institute of Standard and Technology for providing the native biomass materials with which this work was performed. We also gratefully thank Ms. Alexa Schwartz for her various laboratory contributions in assisting with the execution of multiple analyses. We would also like to thank Dr. Peter DeWitt for his review of the statistics used in this publication. 
Author contributions JBS conceived of the procedure and performed initial validation. KPM improved the method and performed validation analyses. BA acquired gel state and solids state NMR analyses and interpreted data. FAP and ALP performed DNP-enhanced NMR measurements. YZ performed and interpreted Raman spectroscopy analysis. WM conducted HPLC-MS experiments and interpreted data. EJW supervised the overall project and edited the manuscript.

Funding Funding provided primarily by U.S. Department of Energy Office of Energy Efficiency and Renewable Energy Bioenergy Technologies Office. DNP-enhanced solid-state NMR experiments were supported by the U.S. Department of Energy, Office of Basic Energy Sciences, Division of Chemical Sciences, Geosciences, and Biosciences. The views expressed in the article do not necessarily represent the views of the DOE or the U.S. Government. The U.S. Government retains and the publisher, by accepting the article for publication, acknowledges that the U.S. Government retains a nonexclusive, paid-up, irrevocable, worldwide license to publish or reproduce the published form of this work, or allow others to do so, for U.S. Government purposes. This work was authored in part by the National Renewable Energy Laboratory, operated by Alliance for Sustainable Energy, LLC, for the U.S. Department of Energy (DOE) under Contract No. DE-AC3608GO28308 and in part by Ames Laboratory operated by Iowa State University under Contract No. DE-AC02-07CH11358.

Data availability All relevant data are within the paper and the Supplementary Information files.

Code availability Not available.

\section{Compliance with ethical standards}

Conflict of interest The authors declare that they have no conflict of interest.

Open Access This article is licensed under a Creative Commons Attribution 4.0 International License, which permits use, sharing, adaptation, distribution and reproduction in any medium or format, as long as you give appropriate credit to the original author(s) and the source, provide a link to the Creative Commons licence, and indicate if changes were made. The images or other third party material in this article are included in the article's Creative Commons licence, unless indicated otherwise in a credit line to the material. If material is not included in the article's Creative Commons licence and your intended use is not permitted by statutory regulation or exceeds the permitted use, you will need to obtain permission directly from the copyright holder. To view a copy of this licence, visit http://creativecommons.org/licenses/by/4.0/.

\section{References}

AOAC International (2012) Method 996.11: starch (total) in cereal products. Official methods of analysis of AOAC
International, 19th edn. The Association, Gaithersburg, MD

Barnes A, De Paëpe G, van der Wel P et al (2008) High-field dynamic nuclear polarization for solid and solution biological NMR. Appl Magn Reson 34:237-263. https://doi. org/10.1007/s00723-008-0129-1

Bax A, Freeman R, Frenkiel TA (1981) An NMR technique for tracing out the carbon skeleton of an organic molecule. J Am Chem Soc 103(8):2102-2104. https://doi.org/10. 1021/ja00398a044

Busse-Wicher M, Gomes TCF, Tryfona T, Nikolovski N, Stott K, Grantham NJ, Bolam DN, Skaf MS, Dupree P (2014) The pattern of xylan acetylation suggests xylan may interact with cellulose microfibrils as a twofold helical screw in the secondary plant cell wall of Arabidopsis thaliana. Plant J 79:492-506. https://doi.org/10.1111/tpj. 12575

Butler H, Ashton L, Bird B et al (2016) Using Raman spectroscopy to characterize biological materials. Nat Protoc 11:664-687. https://doi.org/10.1038/nprot.2016.036

Cheng H, Zhan H, Fu S, Lucia LA (2010) Alkali extraction of hemicellulose from depithed corn stover and effects on soda-AQ pulping. Bioresources 11(1):196-206

Department of Energy (2016) DOE/EE-1436, biomass conversion: from feedstocks to final products. http://Energy.gov/ sites/prod/files/2016/07/f33/conversion_factsheet.pdf. Accessed 14 Oct 2020

Dupree R, Simmons TJ, Mortimer JC, Patel D, Iuga D, Brown SP, Dupree P (2015) Probing the molecular architecture of arabidopsis thaliana secondary cell walls using two- and three-dimensional $13 \mathrm{c}$ solid state nuclear magnetic resonance spectroscopy. Biochemistry 54(14):2335-2345. https://doi.org/10.1021/bi501552k

Eerlingen RC, Delcour JA (1995) Formation, analysis, structure and properties of type III enzyme resistant starch. J Cereal Sci 22(2):129-138. https://doi.org/10.1016/07335210(95) $90042-\mathrm{X}$

Environmental Protection Agency (2014) Regulation of fuels and fuel additives: RFS pathways II, and technical amendments to the RFS STANDARDS and E15 misfueling mitigation requirements. Federal Register. https:// www.federalregister.gov/documents/2014/07/18/2014-16413/ regulation-of-fuels-and-fuel-additives-rfs-pathways-iiand-technical-amendments-to-the-rfs-standards. Accessed 5 June 2020

Environmental Protection Agency (2019) Renewable fuel standard program. https://www.epa.gov/renewable-fuelstandard-program. Accessed 03 June 2020

Isogai A, Atalla RH (1998) Dissolution of cellulose in aqueous $\mathrm{NaOH}$ solutions. Cellulose 5:309-319

Kang X, Kirui K, Dickwella Widanage MC, Mentink-Vigier F, Cosgrove DJ, Wang T (2019) Lignin-polysaccharide interactions in plant secondary cell walls revealed by solidstate nmR. Nat Commun 10(1):347. https://doi.org/10. 1038/s41467-018-08252-0

Kang X, Zhao W, Dickwella Widanage MC, Kirui A, Ozdenvar U, Wang T (2020) CCMRD: a solid-state NMR database for complex carbohydrates. J Biomol NMR 74:239-245. https://doi.org/10.1007/s10858-020-00304-2

Kirui A, Ling Z, Kang X, Dickwella Widanage MC, MentinkVigier F, French AD, Wang T (2019) Atomic resolution of 
cotton cellulose structure enabled by dynamic nuclear polarization solid-state NMR. Cellulose 26(1):329-339. https://doi.org/10.1007/s10570-018-2095-6

Knill CJ, Kennedy JF (2003) Degradation of cellulose under alkaline conditions. Carbohydr Polym 51(3):281-300. https://doi.org/10.1016/S0144-8617(02)00183-2

Lesage A, Bardet M, Emsley L (1999) Through-bond carbon-carbon connectivities in disordered solids by NMR. J Am Chem Soc 121(47):10987-10993. https://doi.org/10. 1021/ja992272b

Li J, Vasanthan T, Gao J, Naguleswaran S, Zijlstra RT, Bressler DC (2014) Resistant starch escaped from ethanol production: evidence from confocal laser scanning microscopy of distiller's dried grains with solubles (DDGS). Cereal Chem 91:130-138. https://doi.org/10.1094/CCHEM-05-130087-R

Maly T, Debelouchina GT, Bajaj VS, Hu KN, Joo CG, MakJurkauskas ML, Sirigiri JR et al (2008) Dynamic nuclear polarization at high magnetic fields. J Chem Phys 128(5):052211. https://doi.org/10.1063/1.2833582

McCleary BV, Charmier LMJ, McKie VA (2019) Measurement of starch: critical evaluation of current methodology. Starch-Stärke 71:1800146. https://doi.org/10.1002/star. 201800146

Murray J (2020) NIST 2019 interlaboratory study to determine starch content in corn grain biomass intermediates. NIST Interagency/Internal Report (NISTIR) - 8291. https://doi. org/10.6028/NIST.IR.8291

Orts WJ, McMahan CM (2016) Biorefinery developments for advanced biofuels from a sustainable array of biomass feedstocks: survey of recent biomass conversion research from agricultural research service. Bioenerg Res 9:430-446. https://doi.org/10.1007/s12155-016-9732-4

Perras FA, Luo H, Zhang X, Mosier NS, Pruski M, Abu-Omar MM (2017) Atomic-level structure characterization of biomass pre- and post-lignin treatment by dynamic nuclear polarization-enhanced solid-state NMR. J Phys Chem A 121(3):623-630. https://doi.org/10.1021/acs.jpca.6b11121

Qi H, Chang C, Zhang L (2008) Effects of temperature and molecular weight on dissolution of cellulose in $\mathrm{NaOH} /$ urea aqueous solution. Cellulose 15:779-787. https://doi.org/ 10.1007/s10570-008-9230-8

Rossini A, Zagdoun A, Hegner F, Schwarzwälder M, Gajan D, Copéret C, Lesage A, Emsley L (2012) Dynamic nuclear polarization NMR spectroscopy of microcrystalline solids. J Am Chem Soc 134(40):16899-16908. https://doi.org/10. 1021/ja308135r
Sajilata M, Singhal RS, Kulkarni PR (2006) Resistant starch-a review. Compr Rev Food Sci Food Saf 5:1-17. https://doi. org/10.1111/j.1541-4337.2006.tb00076.x

Simmons TJ, Mortimer JC, Bernardinelli OD et al (2016) Folding of xylan onto cellulose fibrils in plant cell walls revealed by solid-state NMR. Nat Comm 7(1):13902. https://doi.org/10.1038/ncomms 13902

Slichter CP (2014) The discovery and renaissance of dynamic nuclear polarization. Rep Prog Phys 77(7):072501. https:// doi.org/10.1088/0034-4885/77/7/072501

Sluiter A, Hames B, Hyman D, Payne C, Ruiz R, Scarlata C, Sluiter J, Templeton D, Wolfe J (2008) Determination of total solids in biomass and total dissolved solids in liquid process samples. NREL Laboratory Analytical Procedure. www.nrel.gov/bioenergy/biomass-compositional-analysis. html

Sluiter A, Hames B, Ruiz R, Scarlata C, Sluiter J, Templeton D, Crocker D (2012) Determination of structural carbohydrates and lignin in biomass. NREL Laboratory Analytical Procedure. www.nrel.gov/bioenergy/biomass-compositionalanalysis.html

Solomon BD, Barnes JR, Halvorsen KE (2007) Grain and cellulosic ethanol: history, economics, and energy policy. Biomass Bioenerg 31(6):416-425

Srichuwong S, Jane J (2011) Methods for characterization of residual starch in distiller's dried grains with solubles (DDGS). Cereal Chem 88:278-282. https://doi.org/10. 1094/CCHEM-11-10-0155

Takahashi H, Lee D, Dubois L, Bardet M, Hediger S, De Paëpe $\mathrm{G}$ (2012) Rapid natural-abundance $2 \mathrm{D}{ }^{13} \mathrm{C}-{ }^{13} \mathrm{C}$ correlation spectroscopy using dynamic nuclear polarization enhanced solid-state NMR and matrix-free sample preparation. Angew Chem Int Ed 51(47):11766-11769. https://doi.org/ 10.1002/ange.201206102

Viger-Gravel J, Lan W, Pinon AC, Berruyer P, Emsley L, Bardet M, Luterbacher J (2019) Topology of pretreated wood fibers using dynamic nuclear polarization. J Phys Chem C 123(50):30407-30415. https://doi.org/10.1021/acs.jpcc. $9 \mathrm{~b} 09272$

Zhao W, Fernando LD, Kirui A, Deligey F, Wang T (2020) Solid-state NMR of plant and fungal cell walls: a critical review. Solid State Nucl Magn Reson 107:101660. https:// doi.org/10.1016/j.ssnmr.2020.101660

Publisher's Note Springer Nature remains neutral with regard to jurisdictional claims in published maps and institutional affiliations. 